Canadian

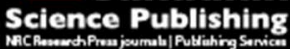

Applied Physiology, Nutrition, and Metabolism Physiologie appliquée, nutrition et métabolisme

\title{
Sorbitol increases muscle glucose uptake ex vivo and inhibits intestinal glucose absorption ex vivo and in normal and type 2 diabetic rats
}

\begin{tabular}{|r|l|}
\hline Journal: & Applied Physiology, Nutrition, and Metabolism \\
\hline Manuscript ID & apnm-2016-0433.R2 \\
\hline Manuscript Type: & Article \\
\hline Complete List of Authors: & $\begin{array}{l}\text { Chukwuma, Chika I.; University of KwaZulu-Natal, Department of } \\
\text { Biochemistry } \\
\text { Islam, Md. Shahidul; University of KwaZulu-Natal }\end{array}$ \\
\hline Keyword: & $\begin{array}{l}\text { Sorbitol, Intestinal glucose absorption, Muscle glusoe uptake, Gastric } \\
\text { emptying, Type 2 diabetes }\end{array}$ \\
\hline \multicolumn{2}{|c}{} \\
\hline
\end{tabular}




\title{
Sorbitol increases muscle glucose uptake ex vivo and inhibits intestinal glucose absorption ex vivo and in normal and type 2 diabetic rats
}

\author{
Chika Ifeanyi Chukwuma and Md. Shahidul Islam* \\ Department of Biochemistry, School of Life Sciences, University of KwaZulu-Natal (Westville \\ Campus), Durban 4000, South Africa
}

*Corresponding author:

Prof. Md. Shahidul Islam, Department of Biochemistry, School of Life Sciences, University of KwaZulu-Natal (Westville Campus), Durban 4000, South Africa. Tel: +27 31260 8717, Fax: +27 31260 7942, E-mail: islamd@ukzn.ac.za

\section{Running title:}

Sorbitol and glucose absorption 


\section{List of abbreviations: (alphabetical)}

DBC, diabetic control

DSO, diabetic sorbitol

GAI, glucose absorption index

GIT, gastrointestinal tract

$\mathrm{GU}_{50}$, concentration to cause $50 \%$ increase of glucose uptake

$\mathrm{IC}_{50}$, concentration to make $50 \%$ inhibition

$\mathrm{NC}$, normal control

NSO, normal sorbitol

ppm, parts per million

T2D, type 2 diabetes 
Abstract: Previous studies have suggested that sorbitol, a known polyol sweetener possesses glycemic control potentials. However, the effect of sorbitol on intestinal glucose absorption and muscle glucose uptake still remains elusive. The present study investigated the effects of sorbitol on intestinal glucose absorption and muscle glucose uptake as possible anti-hyperglycemic or glycemic control potentials using ex vivo and in vivo experimental models. Sorbitol (2.5\% to $20 \%$ ) inhibited glucose absorption in isolated rat jejuna $\left(\mathrm{IC}_{50}=14.6 \pm 4.6 \%\right)$ and increased glucose uptake in isolated rat psoas muscle with $\left(\mathrm{GU}_{50}=3.5 \pm 1.6 \%\right)$ or without insulin $\left(\mathrm{GU}_{50}=\right.$ $7.0 \pm 0.5 \%$ ) in a concentration-dependent manner. Furthermore, sorbitol significantly delayed gastric emptying, accelerated digesta transit, inhibited intestinal glucose absorption and reduced blood glucose increase in both normoglycemic and type 2 diabetic rats after 1 hour of coingestion with glucose. Data of this study suggest that sorbitol exhibited anti-hyperglycemic potentials, possibly via increasing muscle glucose uptake ex vivo and reducing intestinal glucose absorption in normal and type 2 diabetic rats. Hence, sorbitol may be further investigated as a possible anti-hyperglycemic sweetener.

Keywords: Sorbitol, Intestinal glucose absorption, Muscle glucose uptake, Gastric emptying, Type 2 diabetes, Rats 


\section{Introduction}

Diabetes is one of the major global public health problems and its prevalence is rapidly increasing in all over the world, particularly in the developing nations. According to the International Diabetes Federation, about415 million people have diabetes worldwide and this figure is likely to become 642 million by 2040 (International Diabetes Federation 2015). Among two major types of diabetes, type 2 diabetes (T2D) is the most prevalent one, which accounts more than $90 \%$ of all diabetic cases (Loghmani 2005). T2D is a heterogeneous disorder characterized by insulin resistance followed by partial pancreatic beta-cell dysfunction (Partley 2013). It is strongly influenced by sedentary life style, high fat and high calorie diet intake as well as routine consumption of refined and simple carbohydrates e.g. sucrose, fructose and so on (Uusitupa 2002; Hu 2003; Montonen et al. 2007; Stanhope et al. 2011).

Excessive dietary intake of sucrose- and fructose-containing foods and food products has been consistently associated with detrimental health outcomes, such as elevated food intake, body weight gain, visceral adiposity, reduced insulin sensitivity, lipid dysregulation and the risk of developing T2D (Thorburn et al. 1989; Astrup et al. 2002; Elliott et al. 2002; Stanhope et al. 2011).Therefore, there has been an upsurge on the use of alternative sweetening agents, such as sugar alcohols.

Sugar alcohol is one of the classes of sugar substitute that have gained great popularity, especially for overweight, obese and diabetic individuals due to their sweetening ability with lesser undesired calories compared to sucrose (Islam and Indrajit 2012). Additionally, apart from their minimal effect on the integrated blood glucose level compared to sucrose (Talbot and Fisher 1978; Livesey 2003), most commonly used sugar alcohols have been demonstrated to be useful in oral health care (Mäkinen 2010; Gupta et al. 2013).Sorbitol is one of the widely used 
sugar alcohols. Several studies have reported that sorbitol possesses glycemic control effects, but may also possess anti-hyperglycemic effects (Brunzell 1978; Livesey 2003). In a previous study, it has been reported that a $35 \mathrm{~g}$ of orally administered sorbitol caused significantly lower increment in plasma glucose compared to sucrose or fructose in both normal and diabetic human subjects (Akgün and Ertel 1980). In a very recent study, it has been reported that some commonly used sugar alcohols including sorbitol exhibited in vitro alpha glucosidase, alpha amylase and sucrase inhibitory activities (Kang et al. 2014). Additionally, after the evaluation of several studies, the Joint FAO/WHO Expert Committee on Food Additives (JECFA)in a final comment or remark reported that sorbitol may inhibit glucose absorption as well (JECFA 1987).

Although some previous and recent studies suggested the glycemic control or antihyperglycemic potentials of sorbitol in vivo and $\alpha$-glucosidase, $\alpha$-amylase and sucrase inhibitory activities in vitro, the effect of sorbitol on intestinal glucose absorption and muscle glucose uptake still remains unknown. Hence, the present study was conducted to examine the effects of sorbitol on intestinal glucose absorption and gastric emptying as well as muscle glucose uptake as possible anti-hyperglycemic mode of actions by using some ex vivo and in vivo experimental models.

\section{Materials and methods}

\section{Chemicals and reagents}

Streptozotocin, di-basic sodium phosphate, citric acid, adenosine 5'-triphosphate disodium salt hydrate (ATP), D-mannitol, acarbose and sorbitol were purchased from Sigma Aldrich, Germany. Mono-basic sodium phosphate, sodium hydroxide, sodium bicarbonate, sodium chloride, potassium chloride, calcium chloride di-hydrate, mono-basic potassium phosphate, 
magnesium sulphate, sodium hydrogen carbonate, phenol red, trichloacetic acid (TCA), sodium citrate and sodium potassium tartrate were purchased from Merck, South Africa. Glucose and fructose were purchased from Associated Chemical Enterprise (ACE), South Africa, while metformin and Novo rapid insulin were purchased from a local pharmacy store (Pharmed) in Durban, South Africa.

\section{Ex-vivo study}

\section{Animals}

Five adult male Sprague-Dawley rats with mean body weight $183.20 \pm 5.12 \mathrm{~g}$ were procured from the Biomedical Resource Unit (BRU) located at the Westville Campus of the University of KwaZulu-Natal, Durban, South Africa. The animals were fasted over-night (12 hours) and euthanized by halothane anaesthesia. Then the abdominal wall was dissected and the whole gastrointestinal tract (GIT) was collected immediately. Also the entire psoas muscle was collected immediately without causing any damage to the psoas muscle tissue. Intestinal and psoas muscle tissue were immediately used for glucose absorption and glucose uptake study, respectively. All animal procedures were carried out according to the rules and regulations of the Animal Research Ethics Committee of the University of KwaZulu-Natal, Durban, South Africa (Ethical approval number: 092/14/Animal).

\section{Measurement of glucose absorption in isolated rat jejunum}

The time-dependent reduction of glucose concentration in an incubation solution containing $5 \mathrm{~cm}$ of freshly isolated rat jejunum and different sorbitol concentrations $(2.5 \%$ to $20 \%)$ was measured using a method reported by Hassan et al. (2010), with slight modification. Briefly, a 5 
$\mathrm{cm}$ of jejunal segment from the isolated rat GIT was first inverted to expose the villi and then incubated (Steri-Cult $\mathrm{CO}_{2}$ incubator, Labotec, South Africa) in $8 \mathrm{~mL}$ of Kreb's buffer (118 $\mathrm{mmol} / \mathrm{L} \mathrm{NaCl}, 5 \mathrm{mmol} / \mathrm{L} \mathrm{KCl}, 1.328 \mathrm{mmol} / \mathrm{L} \mathrm{CaCl} 2 \cdot 2 \mathrm{H}_{2} \mathrm{O}, 1.2 \mathrm{mmol} / \mathrm{L} \mathrm{KH}_{2} \mathrm{PO}_{4}, 1.2 \mathrm{mmol} / \mathrm{L}$ $\mathrm{MgSO}_{4}$ and $\left.25 \mathrm{mmol} / \mathrm{L} \mathrm{NaHCO}_{3}\right)$ containing $11.1 \mathrm{mmol} / \mathrm{L}(199.98 \mathrm{mg} / \mathrm{dL})$ glucose and increasing concentrations of sorbitol $(2.5 \%$ to $20 \%)$ under an incubation condition of $5 \% \mathrm{CO}_{2}$, 95\% oxygen and $37^{\circ} \mathrm{C}$. Glucose with Kreb's buffer, but without sorbitol was used as a control. Glucose concentrations were measured by using an Automated Chemistry Analyser (LabmaxPlenno, Labtest Inc., Lagoa Santa, Brazil) in all solutions before and after a 2 hours incubation period. The intestinal glucose absorption was calculated as the amount of glucose (mg) absorbed per cm of rat jejunum using the following formula:

$$
\text { Intestinal glucose absorption }=\frac{(\mathrm{GC} 1-\mathrm{GC} 2)}{5 \mathrm{~cm} \text { of jejunum }}
$$

Where, GC1 and GC2 are glucose concentrations (mg/dL) before and after the incubation, respectively.

Percentage glucose absorption inhibition to determine the $\mathrm{IC}_{50}$ value was calculated as follows:

$$
\text { Inhibition }(\%)=\frac{(\Delta \mathrm{GC} \text { for control }-\Delta \mathrm{GC} \text { for sample })}{\Delta \mathrm{GC} \text { for control }} \times 100 \quad \text { (Eq. 2) }
$$

Where $\Delta \mathrm{GC}=\mathrm{GC} 1-\mathrm{GC} 2$.

\section{Type of muscle tissue and viability assay}

Rat psoas muscle (hip flexor) is composed of type I and type II (IIA, IIX and IIB) muscle fibres, with more than $95 \%$ composition of type II, predominantly IIB ( $>60 \%$ ) (Eng et al. 2008), thus 
was suitable for this ex vivo study. The viability of isolated psoas muscle tissue during 1 hour incubation period was confirmed according to previously described methods (Best 2008; Pichugin et al. 2006). These include estimating cellular ATP concentrations and cellular $\mathrm{K}^{+} / \mathrm{Na}^{+}$ ratio of freshly isolated muscle tissue and 1 hour after the incubation at rest in Kreb's buffer under similar experimental incubation conditions as used for ex vivo muscle glucose uptake study. ATP concentration $(\mu \mathrm{M})$ was measured in tissue homogenate using CellTiter-Glo® Luminescent Cell Viability Assay kit (Promega Corporation, Madison, USA). Briefly, a 0.5 g of muscle tissue was homogenized in $5 \mathrm{ml}$ of ice cold PBS and centrifuge at 12,000 $\mathrm{g}$ for $5 \mathrm{~min}$ at $4^{\circ} \mathrm{C}$. Thereafter, $90 \mu \mathrm{l}$ of supernatant or ATP standard solution (3 to $30 \mu \mathrm{M}$ ) was mixed with 90 $\mu 1$ of ATP assay reagent and incubated for $10 \mathrm{~min}$ at $25^{\circ} \mathrm{C}$. Luminescence was measured (Synergy HTX Multi-mode reader, BioTek Instruments Inc, Winooski, USA) and tissue ATP concentration $(\mu \mathrm{M})$ was calculated from an ATP standard curve. On the other hand cellular $\mathrm{K}^{+} / \mathrm{Na}^{+}$ratio in muscle tissue was measured according to a previously reported method with slight modifications (Pichugin et al. 2006). Briefly, a $0.5 \mathrm{~g}$ of tissue (reduced into tiny portions of approximately $1 \mathrm{~mm}$ thick) was first washed twice in $10 \mathrm{ml}$ of $0.3 \mathrm{M}$ D-mannitol solution by a 2 min gentle agitation to remove ions from the extracellular spaces of the tiny slices. Thereafter, the washed tissue was incubated in $20 \mathrm{ml}$ of $3 \% \mathrm{w} / \mathrm{v}$ trichloroacetic acid (TCA) for $24 \mathrm{~h}$ to rupture cells of tissue and release intracellular ions. The $\mathrm{K}^{+}$and $\mathrm{Na}^{+}$concentrations (ppm) were measured in the TCA supernatant using a flame photometer (Jenway PFP7 Flame Photometer, Jenway Ltd., Felsted, UK).

\section{Measurement of glucose uptake in isolated rat psoas muscles}


The effect of sorbitol on glucose uptake in isolated rat psoas muscles was determined according to the procedures modified from a previously reported method (Hassan et al. 2010), by monitoring the glucose concentration change in an incubation solution containing $0.5 \mathrm{~g}$ of freshly isolated rat psoas muscle and different sorbitol concentrations. Briefly, a $0.5 \mathrm{~g}$ of isolated rat psoas muscle was incubated in $8 \mathrm{~mL}$ of Kreb's buffer containing $11.1 \mathrm{mmol} / \mathrm{L}$ (199.98 mg/dL) glucose and increasing concentrations of sorbitol $(2.5 \%$ to $20 \%$ )or $2 \mathrm{mg} / \mathrm{mL}$ metformin (met, as positive control) with and without $200 \mathrm{mU} / \mathrm{mL}$ insulin, under a $5 \% \mathrm{CO}_{2}, 95 \%$ oxygen and $37{ }^{\circ} \mathrm{C}$ incubation condition. Glucose with Kreb's buffer, but without sorbitol was used as a control. Glucose concentration was measured in all incubated solutions before and after 1 hour incubation and the muscle glucose uptake was calculated as the amount of glucose (mg) absorbed per gram of rat psoas muscle using the following formula:

$$
\text { Muscle glucose uptake }=\frac{(\mathrm{GC} 1-\mathrm{GC} 2)}{0.5 \mathrm{~g} \text { of muscle tissue }}
$$

Where, GC1 and GC2 are glucose concentrations (mg/dL) before and after the incubation, respectively.

Percentage glucose uptake used to determine the concentration of sorbitol required to cause $50 \%$ increase of muscle glucose uptake $\left(\mathrm{GU}_{50}\right)$, with or without insulin was calculated as per following formula:

$$
\% \text { increase in glucose uptake }=\frac{(\Delta \mathrm{GC} \text { for sample }-\Delta \mathrm{GC} \text { for control })}{\Delta \mathrm{GC} \text { for control }} \times 100
$$

Where $\Delta \mathrm{GC}=\mathrm{GC} 1-\mathrm{GC} 2$

\section{In vivo study}

\section{Animals}


Twenty-eight seven-week-old male Sprague-Dawley (SD) rats with mean body weight $179.14 \pm 9.12 \mathrm{~g}$ were procured from the Biomedical Resource Unitlocated at the Westville Campus of the University of KwaZulu-Natal, Durban, South Africa. Animals were randomly divided into five groups, with five animals in each normal group and six animals in each diabetic group. The groups were namely: normal control (NC), normal sorbitol (NSO), diabetic control (DBC), diabetic sorbitol (DSO) and diabetic acarbose (DBA). All animals were fed with a commercial rat pellet diet (composition: $13 \%$ protein, 35\% starch, 2.5 fat, $7 \%$ fibre, and vitamin and mineral premix, energy: $2.464 \mathrm{kcal} / \mathrm{g}$, Meadow Feeds KwaZulu-Natal, Pietermaritzburg, South Africa) and were maintained according to the rules and regulations of the Experimental Animal Ethics Committee of the University of KwaZulu-Natal, South Africa during the entire experimental period (Ethical approval number: 092/14/Animal).

\section{Induction of diabetes}

Insulin resistance and partial pancreatic $\beta$-cell dysfunction are the two major etiological and pathophysiological characteristics of T2D. In order to induce T2D, we adopted the methods previously reported by Wilson and Islam (2012).Briefly, a 10\% fructose solution was supplied in the form of drinking water for the first two weeks to the animals in diabetic groups (DBC, DSO and DBA) to induce insulin resistance, while the animals in the NC and NSO groups were supplied with normal drinking water. Thereafter, animals in DBC, DSO and DBA groups were injected with a single dose (i.p.) of streptozotocin $(40 \mathrm{mg} / \mathrm{kg}$ body weight) dissolved in citrate buffer ( $\mathrm{pH} 4.5)$ to induce partial pancreatic $\beta$-cell dysfunction, whereas the animals in the NC and NSO groups were injected with citrate buffer only. One week after the streptozotocin injection, non-fasting blood glucose levels of all animals was measured using a portable 
Glucometer (Glucoplus Inc., Saint-Laurent, Quebec, Canada), and rats with blood glucose level $>300 \mathrm{mg} \mathrm{dL}^{-1}$ were considered as diabetic, and used for the study, while those with blood glucose levels $<300 \mathrm{mg} \mathrm{dL}^{-1}$ were excluded from the study.

\section{Feeding and sampling}

After the confirmation of diabetes, all animals were fasted overnight (16 hours) with free access to drinking water only and fasting blood glucose $(\mathrm{mg} / \mathrm{dL})$ was measured using a portable glucometer (Glucoplus Inc., Saint-Laurent, Quebec, Canada). Thereafter, the animals in the NSO and DSO groups were administered with an oral bolus dose of sorbitol ( $0.4 \mathrm{~g} / \mathrm{kg}$ body weight $)$ with glucose ( $2 \mathrm{~g} / \mathrm{kg}$ body weight), while animals in the DBA group were administered with an oral bolus dose of acarbose (100 mg/kg body weight) with glucose ( $2 \mathrm{~g} / \mathrm{kg}$ body weight). Oral bolus dose of sorbitol was chosen considering the tolerance of sorbitol after ingestion (Schiweck and Ziesenitz 1996; Livesey 2003), while acarbose was used as a positive control, because it is a standard anti-diabetic drug that controls postprandial hyperglycemia. On the other hand, only glucose (2 $\mathrm{g} / \mathrm{kg}$ body weight) was administered to the animals in NC and DBC groups. All administrations contained $0.05 \%$ phenol red as a recovery marker. Exactly one hour after the ingestion, while the animals had no access to food or drinking water, they were sacrificed using halothane anaesthesia. Blood sample (using cardiac puncture) as well as the gastro-intestinal tract (GIT) of each animal was quickly collected. Glucose concentration was measured in the blood samples using an Automated Chemistry Analyzer (LabmaxPlenno, Labtest, Lagoa Santa, Brazil).The GIT from each animal was quickly removed, frozen immediately in liquid nitrogen to prevent the movement of the contents, and preserved immediately at $-30^{\circ} \mathrm{C}$ for further analysis. 


\section{Sample preparation}

Each GIT was thawed and divided into eight segments: stomach; 1st, 2nd, 3rd, and 4th quarters of small intestine; cecum; proximal and distal half of the colon. Content weight of each segment was determined by subtracting the weight of the segment without content from the respective weight of the segment with content. Contents and tissues were collected and individually homogenized in ice cold normal saline (Ultra Turrax Tube Drive Work Station homogenizer, IKA Laboratory equipment, Staufen, Germany) and centrifuged twice at 15,000 rpm for 30 min (HettichMikro 200 microcentrifuge, Hettich Lab Technology, Tuttlingen, Germany) as reported previously (Chukwuma and Islam 2015). Phenol red concentration was determined spectrophotometrically (Synergy HTX Multi-mode reader, BioTek Instruments Inc, Winooski, USA) with bile acid correction in the supernatants of contents and tissue segments according to a previously published method (Chukwuma and Islam 2015). Briefly, a $30 \mu 1$ of supernatant or phenol red standard (concentrations $0.0038 \%-0.00025 \%$ ) was mixed with $210 \mu 1$ of $0.1 \mathrm{mmol} / \mathrm{L}$ dibasic sodium phosphate solution $(\mathrm{pH} \mathrm{10.5).} \mathrm{Then} \mathrm{the} \mathrm{optical} \mathrm{density} \mathrm{at} 420 \mathrm{~nm}$ was subtracted from the optical density at $620 \mathrm{~nm}$ (for bile acid correction) to obtain the final optical density. The concentration as well as the amount of recovered phenol red was calculated from the standard curve. Glucose concentration in the intestinal contents was measured using an Automated Chemistry Analyzer (LabmaxPlenno, Labtest Inc., Costa Brava, Brazil) using commercial assay kits.

\section{Calculations}


Glucose $(\mathrm{g})$ and phenol red $(\mathrm{g})$ recovered from each GIT segment was calculated and used as indexes to calculate gastric emptying, glucose absorption index (GAI) and digesta transit according to previously described methods (Islam and Sakaguchi 2006; Chukwuma and Islam 2015).

Gastric emptying, denoting the emptying time of stomach content was calculated using the following formula:

$$
\text { Gastric emptying }(\%)=\frac{\mathrm{A}-\mathrm{B}}{\mathrm{A}} \times 100
$$

Where, "A" is the total amount of phenol red $(\mathrm{g})$ recovered from GIT; and " $\mathrm{B}$ " is the total amount of phenol red $(\mathrm{g})$ recovered from the stomach.

GAI denotes the degree of glucose absorption in each segment of GIT. It is the percentage amount of the glucose absorbed passing through a given segment of GIT, and was calculated using the following formula:

$$
\text { Glucose absorption index (\%) in a given segment of GIT }=1-\frac{\frac{a}{b}}{\frac{c}{d}} X 100
$$

Where, "a" is the amount of glucose (g) recovered from that segment; "b" is the amount of phenol red $(\mathrm{g})$ recovered from the same segment; "c" is the amount of glucose (g) given to corresponding animal; and " $\mathrm{d}$ " is the amount of phenol red (g) given to the corresponding animal. 
Digesta transit in a particular segment of the intestine is the ratio of the amount of content leaving that segment to the amount reaching the same segment. It was calculated in percentage using the following formula:

$$
\text { Digesta transit in a given segment }(\%)=\frac{\mathrm{a}}{\mathrm{b}} \times 100
$$

Where "a" is the amount of phenol red $(\mathrm{g})$ recovered from that particular segment of the GIT to the distal colon excluding the amount of phenol red $(\mathrm{g})$ recovered from that particular segment and " $b$ " is the amount of phenol red (g) recovered from that particular segment of the GIT to the distal colon.

\section{Results}

\section{Ex vivo study}

\section{Effect of sorbitol on glucose absorption on isolated rat jejunum}

Data showing the effects of sorbitol on glucose absorption in isolated rat jejunum are presented in Fig. 1. The data showed a concentration-dependent effect of sorbitol on jejunal glucose absorption ex vivo. Incubation of jejunal tissue in $5 \%, 10 \%$ and $20 \%$ sorbitol significantly reduced glucose absorption $(\mathrm{p}<0.05)$ compared to the control, when no significant difference was observed between the control and 2.5\% sorbitol containing sample. Additionally, no significant difference was observed between the 5\%,10\% and $20 \%$ sorbitol containing samples, while glucose absorption at $20 \%$ sorbitol concentration was significantly reduced $(\mathrm{p}<0.05)$ compared to glucose absorption at $2.5 \%$ sorbitol concentration (Fig. 1).

\section{Effect sorbitol on glucose uptake by isolated rat psoas muscle}


The data for the effects of sorbitol on glucose uptake by isolated rat psoas muscle are presented in Fig. 2 and Table 1. Data showed that sorbitol (2.5\% to 20\%) increased dosedependent glucose uptake in the isolated rat psoas muscle with $\left(\mathrm{GU}_{50}=3.53 \pm 1.61 \%\right)$ or without $\left(\mathrm{GU}_{50}=7.02 \pm 0.46 \%\right)$ insulin. Glucose uptake at $10 \%$ and $20 \%$ concentrations of sorbitol was significantly higher $(\mathrm{p}<0.05)$ than the control and $2.5 \%$ sorbitol containing sample. No significant difference in glucose uptake was observed between the $5 \%$ and $20 \%$ sorbitol containing samples with or without insulin (Fig. 2). A $2 \mathrm{mg} / \mathrm{mL}$ metformin significantly increased muscle glucose uptake than all sorbitol containing samples with or without insulin, while incubation with insulin significantly influenced $(p<0.05)$ the glucose uptake effect of sorbitol (Table 1). Similar effects of metformin were found in the previous studies as well (Turban et al. 2012; Kristensen et al. 2014).

Furthermore, $1 \mathrm{~h}$ incubation of psoas muscle tissue did not significantly influence the cellular ATP concentration and $\mathrm{K}^{+} / \mathrm{Na}^{+}$ratio compared to fresh tissue which was used for the muscle glucose uptake study (Table 2).

\section{In vivo study}

\section{Effects of sorbitol on intestinal glucose absorption}

The data for intestinal glucose absorption index (GAI) in the different intestinal segments of the animals in the different groups are presented in Fig. 3. Administration of either sorbitol or acarbose significantly influenced GAI in the different intestinal segments of both normal and diabetic animals. Ingestion of sorbitol significantly reduced $(p<0.05)$ GAI in the $1^{\text {st }}$ and $2^{\text {nd }} q$ trs of the small intestine in both normal and diabetic animals, while ingestion of acarbose significantly reduced $(\mathrm{p}<0.05)$ GAI in the $1^{\text {st }}$ qtr of the small intestine of diabetic animals (Fig. 3$)$. In the 
proximal colon sorbitol and acarbose treatment significantly reduced $(p<0.05)$ GAI in normal and diabetic animals respectively, while no significant difference was observed in the other intestinal segments of different animal groups (Fig. 3)

\section{Effect of sorbitol on gastric emptying and digesta transit}

The data for the effect of sorbitol on gastric emptying and digesta transit are represented in Fig. 4 and Table 3. Gastric emptying significantly increased $(p<0.05)$ after the induction of diabetes but significantly decreased $(p<0.05)$ after the treatment with either sorbitol or acarbose. Significantly slower gastric emptying of diabetic animals was observed in the acarbose ingested group compared to the sorbitol ingested groups. Treatment with sorbitol did not significantly affect gastric emptying in normal animals (Fig. 4).

On the other hand sorbitol treatments significantly increased $(\mathrm{p}<0.05)$ digesta transit from the $3^{\text {rd }}$ qtr of the small intestine up to proximal colon in normal rats (Table 3 ). In diabetic animals however, sorbitol and acarbose treatment only significantly increased $(\mathrm{p}<0.05)$ digesta transit in the cecum and proximal colon, while no significant difference was observed in the other intestinal segments regardless of the animal group or treatment (Table 3).

\section{Effects of sorbitol on blood glucose}

Fig. 5 presents the effects of sorbitol on blood glucose level in treated animals. The single oral dose of sorbitol and acarbose co-ingested with glucose significantly reduced ( $p>0.05)$ blood glucose increase $1 \mathrm{~h}$ after the dose administration in diabetic animals, although the results were not significantly different for normal animals (Fig. 5). 


\section{Discussion and conclusion}

Sugar alcohols are known for their lower caloric value compared to sucrose when sorbitol is widely used due to its number of beneficial effects on health (Hayes 2001; Livesey 2003).It has been speculated that sorbitol may inhibit intestinal glucose absorption (JECFA 1987). A previous suggested that sorbitol may be useful for glycemic control in normoglycemic and diabetic subjects (Akgün and Ertel 1980), when a recent study reported that sorbitol possess inhibitory effect on carbohydrate digesting enzyme activities in vitro (Kang et al. 2014). However, none of these previous studies investigated the effect of sorbitol on intestinal glucose absorption and muscle glucose uptake. Hence, the present study investigated the effects of sorbitol on intestinal glucose absorption and muscle glucose uptake to further understand the mechanisms behind the effects of sorbitol on dietary glucose absorption and uptake.

Dietary glucose is actively absorbed from the intestine, contributing to blood glucose rise, but absorption capacity of glucose varies from one intestinal segment to another. However, it has been reported that majority of dietary glucose is likely to be absorbed from the proximal to mid small intestine (part of the duodenum and jejunum) (Bogner et al. 1963; Rider et al. 1967; Lavin 1976), and for the same reason intestinal tissue from this section was used for the ex-vivo glucose absorption study. In our ex vivo study, the amount of glucose absorbed by the jejunal segments of rats reached a peak value of $5.20 \pm 072 \mathrm{mg} / \mathrm{cm}$ jejunum after 2 hour incubation period in a glucose solution without sorbitol (Fig. 1). However, this peak value was significantly reduced $(\mathrm{p}<0.05)$ to $3.53 \pm 0.50 \mathrm{mg} / \mathrm{cm}$ jejunum in the presence of $5 \%$ sorbitol and reached as low as $2.87 \pm 0.70 \mathrm{mg} / \mathrm{cm}$ jejunum in the presence of $20 \%$ sorbitol, which suggests the possible inhibitory effect of sorbitol on intestinal glucose absorption. 
Previous studies have demonstrated that co-ingestion of glucose with sorbitol increased sorbitol absorption relative to ingestion of sorbitol alone (Beaugerie et al. 1995, 1996).Our in vivo data showed high glucose absorption index (GAI) across the different intestinal segments of rats (Fig. 3), which accounts for the complete and rapid intestinal absorption of glucose. However, the significantly reduced $(\mathrm{p}<0.05)$ GAI in the 1 st and 2 nd qtrs of the small intestine of normal and diabetic rats fed with sorbitol (Fig. 3) correspond to the results of our ex vivo glucose absorption study (Fig. 1) and further confirms the inhibitory potentials of sorbitol on intestinal glucose absorption. In fact, the observed inhibitory effect of sorbitol in the proximal small intestine is of particular interest, because the proximal small intestine is most likely the site where majority of glucose absorption occurs (Rider et al. 1967). In our study, sorbitol significantly inhibited the glucose absorption in this region of small intestine when the effect was comparable to that of a widely used anti-diabetic drug (acarbose) in both normal and diabetic animals (Fig. 3). Additionally, the reduced postprandial blood glucose increase observed in the sorbitol-fed animals (Fig. 5) may also be a reflection of the inhibitory effect of sorbitol on intestinal glucose absorption (Fig. 5). Based on the above discussions we hypothesize that the observed reduced intestinal glucose absorption in sorbitol-fed rats (Fig. 5) might be due to the reported increased intestinal sorbitol absorption when co-ingested with glucose (Beaugerie et al. 1995, 1996). However, this hypothesis requires further investigation to elucidate the actual absorption-related interaction between sorbitol and glucose in the different sections of intestine.

Delayed gastric emptying and accelerated digesta transit can contribute to reduced intestinal nutrient absorption as well as food intake (Salminen 1984; Shafer 1987) and has been suggested to be partly involved in the mode of action of acarbose in reducing intestinal glucose absorption (Ranganath 1998). In our study, the induction of diabetes significantly increased gastric 
emptying rate (Fig. 4), which depicts the clinical presentation of gastric emptying in most type 2 diabetic patients (Phillips et al. 1991; Horowitz et al. 1996. However, an oral bolus dose of sorbitol significantly reduced $(\mathrm{p}<0.05)$ gastric emptying in diabetic rats (Fig. 4$)$ and increased the digesta and colonic transit in both normal and diabetic animals (Table 3). These data are in line with the results of previous studies in healthy human subjects (Hunt 1963; Skoog et al. 2006) and may also contribute to the lower GAI in the proximal half of the small intestine (Fig. 3).

Under physiological condition, the blood glucose homeostasis is constantly maintained by the action of two important hormones: insulin and glucagon. Among other functions, insulin promotes the clearance of circulating glucose for either storage or energy production in cells, thus prevent abnormal increment of blood glucose level (Aronoff et al. 2004).Previous studies have reported the glycogenic effects of oral or parenteral sorbitol administration in rats (Stetten and Stetten 1951), which suggests possibilities of sorbitol to enhance clearance of circulating glucose towards glycogen synthesis. In our present study, sorbitol exhibited a concentrationdependent increase in muscle glucose uptake with or without insulin compared to control (Fig. 2), when insulin significantly enhanced the muscle glucose uptake effect of sorbitol (Table 1) (Turban et al. 2012; Kristensen et al. 2014).This data suggests the potential of sorbitol in improving insulin-mediated muscle glucose uptake at least in ex vivo condition, but still requires further investigation at in vivo level.

In summary, apart from the effects of sorbitol on enhancing muscle glucose uptake ex vivo (Fig. 2 and Table1), the results of our present study suggest that sorbitol possesses inhibitory effects on intestinal glucose absorption ex vivo (Fig. 1) which have been further supported by the results of in vivo study in normal and diabetic rats (Fig. 3 and 5). Additionally, our present study also revealed that the ingestion of sorbitol delayed gastric emptying (Fig. 4) and accelerated 
digesta transit (Table 3) in normal and diabetic rats, which might partly contribute to the observed in vivo inhibitory effects sorbitol on small intestinal glucose absorption.

In conclusion, the data from our present study suggest that sorbitol not only exhibit potential effects on inhibiting intestinal glucose absorption but may also possess potentials of increasing muscle glucose uptake, which both can contribute to glycemic control effects. Hence, sorbitol may be further investigated as a potential anti-hyperglycemic sweetener in diabetic foods and food products.

\section{Acknowledgements}

Special thanks to Dr M. Singh (Biochemistry) to allow us to use her lab and Dr Linda Bester, Erukainure O.L., David Mompe, and Deliwe Mdakane for their technical assistance during this study.

\section{Conflict of interest}

The authors declare that there is no conflict of interest within this article.

\section{References}

Akgün, S. and Ertel, N.H. 1980. A comparison of carbohydrate metabolism after sucrose, sorbitol, and fructose meals in normal and diabetic subjects. Diabetes Care. 3(5): 582-585. doi: 10.2337/diacare.3.5.582.PMID: 7002512 . 
Aronoff, S.L., Berkowitz, K., Shreiner, B., and Want, L. 2004. Glucose metabolism and regulation: beyond insulin and glucagon. Diabetes Spectr. 17: 183-190. doi: 10.2337/diaspect.17.3.183.

Astrup, A., Raben, A., Vasilaras, T.H., and Moller, A.C. 2002. Sucrose in soft drinks is fattening: a randomized 10 week study in overweight subjects. Am. J. Clin. Nutr. 75(2): 405S. Available from http://ajcn.nutrition.org/content/75/2/339S.full.pdf + html [accessed 25 March 2016].

Beaugerie, L., Flourié, B., Lémann, M., Achour, L., Franchisseur, C., and Rambaud, J.C. 1995. Sorbitol absorption in the healthy human small intestine is increased by the concomitant ingestion of glucose or lipids. Eur. J. Gastroenterol. Hepatol. 7: 125-128. PMID: 7712303.

Beaugerie, L., Lémann, M., Jian, R., Flourié, B., Rain, J.D., and Rambaud, J.C. 1996. Effect of glucose and lipids on intestinal absorption of sorbitol: role of gastric emptying. Neurogastroenterol. Motil. 8(3): 235-239. doi: 10.1111/j.1365-2982.1996.tb00262.x. PMID: 8878083.

Best, B.P. 2008. Scientific justification of cryonics practice. Rejuvenation Res. 11(2): 493-503. doi: 10.1089/rej.2008.0661.

Bogner, P.H., Haines, T.A., and McLain, P.L. 1963. Comparison of aerobic and anaerobic active transport by different intestinal regions of young female chicks. Am. Zool. 3(4): 537. doi: 10.1093/icb/3.4.482.

Brunzell, J.D. 1978. Use of fructose, sorbitol, or xylitol as a sweetener in diabetes mellitus. Diabetes Care.1(4): 233-230. doi: 10.2337/diacare.1.4.223. PMID: 400132. 
Chukwuma, C.I., and Islam, M.S. 2015. Effects of xylitol on carbohydrate digesting enzymes activity, intestinal glucose absorption and muscle glucose uptake: a multi-mode study. Food Funct. 6(3): 955-962. doi: 10.1039/c4fo00994k. PMID: 25656339.

Elliott, S.S., Keim, N.L., Stern, J.S., Teff, K., and Havel, P.J. 2002. Fructose, weight gain, and the insulin resistance syndrome. Am. J. Clin. Nutr. 76(5): 911-922. PMID: 12399260.

Eng, C.M., Smallwood, L.H., Rainiero, M.P., Lahey, M., Ward, S.R. and Lieber, R.L. 2008. Scaling of muscle architecture and fiber types in the rat hind limb. J Exp Biol. 211(Pt 14): 2336-2345. doi: 10.1242/jeb.017640.

Gupta, P., Gupta, N., Pawar, A.P., Birajdar, S.S., Natt, A.S., and Singh, H.P. 2013. Role of sugar and sugar substitutes in dental caries: a review. ISRN Dent. 2013: 519421. doi: 10.1155/2013/519421. PMID: 24490079.

Hassan, Z., Yam, M.F., Ahmad, M., and Yusof, A.P. 2010. Anti-diabetic properties and mechanism of action of Gynurapro cumbens water extract in streptozotocin - induced diabetic rats. Molecules 15(12): 9008-9025. doi: 10.3390/molecules15129008. PMID: 21150821.

Hayes, C. 2001. The effect of non-cariogenic sweeteners on the prevention of dental caries: a review of the evidence. J. Dent. Educ. 65(10): 1106-1109. PMID: 11699985.

Horowitz, M., Wishart, J.M., Jones, K.L., and Hebbard, G.S. 1996. Gastric emptying in diabetes: an overview. Diabet. Med. 13(9): S16-22. PMID: 8894465.

Hu, F.B. 2003. Sedentary lifestyle and risk of obesity and type 2 diabetes. Lipids. 38(2): 103108. doi:10.1007/s11745-003-1038-4. PMID: 12733740.

Hunt, J.N. 1963. The duodenal regulation of gastric emptying. Gastroenterol 45: 149-156. PMID: 14051726. 
International Diabetes Federation. 2015. IDF Diabetes Atlas, $7^{\text {th }}$ edition [online]. Available fromhttp://www.diabetesatlas.org/resources/2015-atlas.html [accessed 8 February 2016].

Islam, M.S., and Indrajit, M. 2012. Effects of xylitol on blood glucose, glucose tolerance, serum insulin and lipid profile in a type 2 diabetes model of rats. Ann. Nutr. Metab. 61(1):57-64. doi: 10.1159/000338440. PMID: 22832597.

Islam, M.S., and Sakaguchi, E. 2006. Sorbitol-based osmotic diarrhea: Possible causes and mechanism of prevention investigated in rats. World J. Gastroenterol. 12(47): 7635-7641. doi: 10.3748/wjg.v12.i47.7635. PMID: 17171792.

Joint FAO/WHO Expert Committee on Food Additives. 1987. Food Additives series, No. 20. Available from http://www.inchem.org/documents/jecfa/jecmono/v20je13.htm [accessed 25 March 2016].

Kang Y, Jo S, Yoo J, Cho J, Kim E, Apostolidis E., and Kwon, Y. 2014. Anti-hyperglycemic effect of selected sugar alcohols. FASEB J. 28(2): 829.32. Available from http://www.fasebj.org/content/28/1_Supplement/829.32 [accessed 26 January 2016].

Kristensen, J.M., Treebak, J.T., Schjerling, P., Goodyear, L., Wojtaszewski, J.F. 2014. Two weeks of metformin treatment induces AMPK-dependent enhancement of insulin-stimulated glucose uptake in mouse soleus muscle. Am. J. Physiol. Endocrinol. Metab. 306(10): E1099E1109. doi: 10.1152/ajpendo.00417.2013. Epub 2014 Mar 18. PMID:24644243.

Lavin, R.J. 1976. Digestion and absorption of carbohydrate from embryo to adult. In Digestion in the Fowl. Edited by R.N. Boorman and B.M. Freeman. British Poultry Science Ltd, Edinburgh. pp. 63-116. 
Livesey, G. 2003. Health potential of polyols as sugar replacers, with emphasis on low glycaemic properties. Nutr. Res. Rev. 16(2): 163-191. doi: 10.1079/NRR200371. PMID: 19087388.

Loghmani, E. 2005. Diabetes mellitus: Type 1 and type 2. In Guide lines for adolescent nutrition service. Edited by J. Stang. University of Minnesota, Minneapolis. pp. 167-182. Available from http://www.epi.umn.edu/let/pubs/adol_book.shtm [accessed 21 September 2015].

Mäkinen, K.K. 2010. Sugar alcohols, caries incidence, and remineralization of caries lesions: A literature review. Int. J. Dent. doi: 10.1155/2010/981072. PMID: 20339492.

Montonen, J., Järvinen, R., Knekt, P., Heliövaara, M., and Reunanen, A. 2007. Consumption of sweetened beverages and intakes of fructose and glucose predict type 2 diabetes occurrence. J. Nutr. 137(6): 1447-1454. PMID: 17513405.

Partley, R.E. 2013. The early treatment of type 2 diabetes. Am. J. Med. 126(9): S2-S9. doi: 10.1016/j.amjmed.2013.06.007. PMID: 23953075.

Phillips, W.T., Schwartz, J.G., and McMahan, C.A. 1991. Rapid gastric emptying in patients with early non-insulin-dependent diabetes mellitus. N. Engl. J. Med. 324(2): 130-131. doi: 10.1056/NEJM199101103240217. PMID: 1984184.

Pichugin, Y., Fahy, G.M., and Morin, R. 2006. Cryopreservation of rat hippocampal slices by vitrification. Cryobiology. 52(2): 228-240. doi: 10.1016/j.cryobiol.2005.11.006.

Ranganath, L., Norris, F., Morgan, L., Wright, J., and Marks, V. 1998. Delayed gastric emptying occurs following acarbose administration and is a further mechanism for its antihyperglycaemic effect. Diabet. Med.15(2): 120-124. doi: 10.1002/(SICI)10969136(199802)15:2<120::AID-DIA529>3.0.CO;2-I. PMID: 9507911. 
Rider, A.K., Schedl, H.P., Nokes, G., and Shining, S. 1967. Small intestinal glucose transport. Proximal-distal kinetic gradients. J. Gen. Physiol. 50(5): 1173-1182. doi:10.1085/jgp.50.5.1173. PMID: 6033580.

Salminen, E., Salminen, S., Porkka, L., and Koivistoinen, P. 1984. The effects of xylitol on gastric emptying and secretion of gastric inhibitory polypeptide in the rat. J. Nutr. 114(12): 2201-2203. PMID: 6502263.

Schiweck, H., and Ziesenitz, S.C., 1996. Physiological properties of polyols. In Advances in Sweeteners. Edited by T.H. Grenby. Blackie Academic and Professional, London. pp. 56-84.

Shafer, R.B., Levine, A.S., Marlette, J.M., and Morley, J.E. 1987. Effects of xylitol on gastric emptying and food intake. Am. J. Clin. Nutr. 45(4): 744-747. PMID: 3565302.

Skoog, S.M., Bharucha, A.E., Camilleri, M., Burton, D.D., and Zinsmeister, A.R. 2006. Effects of an osmotically active agent on colonic transit. Neurogastroenterol. Motil. 18(4): 300-306. doi: 10.1111/j.1365-2982.2006.00757.x. PMID: 16553585 .

Stanhope, K.L., Bremer, A.A., Medici, V., Nakajima, K., Ito, Y., Nakano, T., et al. 2011. Consumption of fructose and high fructose corn syrup increase postprandial triglycerides, LDL-cholesterol, and apolipoprotein-B in young men and women. J. Clin. Endocrinol. Metab. 96(10): E1596-1605. doi: 10.1210/jc.2011-1251. PMID: 21849529.

Stetten, M.R., and Stetten, D. 1951. Metabolism of sorbitol and glucose compared in normal and alloxan-diabetic rats. J. Biol. Chem. 193(1): 157-165. PMID: 14907701.

Talbot, J.M., and Fisher, K.D. 1978. The need for special foods and sugar substitutes by individuals with diabetes mellitus. Diabetes Care. 1(4): 231-240. doi: 10.2337/diacare.1.4.231. PMID: 400133. 
Thorburn, A.W., Storlien, L.H., Jenkins, A.B., Khouri, S., and Kraegen, E.W. 1989. Fructoseinduced in vivo insulin resistance and elevated plasma triglyceride levels in rats. Am. J. Clin. Nutr. 49(6): 1155-1163. PMID: 2658534.

Turban, S., Stretton, C., Drouin, O., Green, C.J., Watson, M.L., Gray, A., et al. 2012. Defining the contribution of AMP-activated protein kinase (AMPK) and protein kinase $\mathrm{C}(\mathrm{PKC})$ in regulation of glucose uptake by metformin in skeletal muscle cells. J. Biol. Chem. 287(24): 20088-20099. doi: 10.1074/jbc.M111.330746. Epub 2012 Apr 17. PMID: 22511782.

Uusitupa, M. 2002. Lifestyles matter in the prevention of type 2 diabetes. Diabetes Care 25(9): 1650-1651. Doi: 10.2337/diacare.25.9.1650. PMID: 12196442.

Wilson, R.D., and Islam, M.S. 2012. Fructose-fed streptozotocin-injected rat: an alternative model for type 2 diabetes. Pharmacol. Rep. 64(1): 129-139. doi: 10.1016/S17341140(12)70739-9. PMID: 22580529. 


\section{TABLES}

Table 1. Percentage $\mathrm{GU}_{50}$ values of glucose uptake increase of sorbitol in isolated psoas muscle, with and without insulin.

With insulin $[200 \mathrm{mU} / \mathrm{mL}] \quad$ Without insulin

GU50 [\%]* $3.53 \pm 1.61^{\mathrm{a}} \quad 7.02 \pm 0.46^{\mathrm{b}}$

Data are presented as mean \pm SD of triplicate analysis. ${ }^{\text {ab }}$ Values with different superscript letters are significantly different from each other ( $\mathrm{p}<0.05$; Tukey's HSD multiple range post-hoc test). $* \mathrm{GU}_{50}$ : Concentration ( $\%$ as $\left.\mathrm{w} / \mathrm{v}\right)$ of sorbitol required to cause $50 \%$ increase of muscle glucose uptake. 
Table 2: Verification of psoas muscle tissue viability before and after one hour incubation period.

Psoas muscle tissue viability indexes

ATP level $(\mu \mathrm{M}) \quad \mathrm{K}^{+} / \mathrm{Na}^{+}$ratio viability index

$\mathrm{K}^{+}$conc. (ppm) $\quad \mathrm{Na}^{+}$conc. $(\mathrm{ppm}) \quad \mathrm{K}^{+} / \mathrm{Na}^{+}$ratio

$\begin{array}{lllll}\text { Freshly isolated } & 21.27 \pm 2.46 & 10.27 \pm 0.30 & 1.11 \pm 0.02 & 9.24 \pm 0.30\end{array}$

tissue

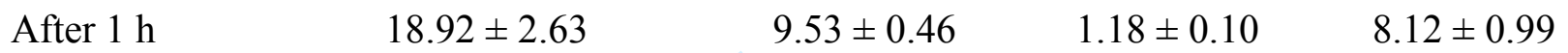

incubation in

Kreb's solution**

**Tissue incubation condition was similar to ex vivo experimental condition $\left(5 \% \mathrm{CO}_{2}, 95 \%\right.$ oxygen and $37^{\circ} \mathrm{C}$ ). ppm: parts per million. 
Table 3. Percentage digesta transit in the different segments of the intestinal tract during a one hour experimental period.

\begin{tabular}{|c|c|c|c|c|c|c|}
\hline \multirow[t]{2}{*}{ GRP } & $1^{\text {st }} \mathrm{qtr}$ & $2^{\text {nd }} q \operatorname{tr}$ & $3^{\text {rd }} \mathrm{qtr}$ & $4^{\text {th }} \mathrm{qtr}$ & \multirow[t]{2}{*}{ Cecum } & Prox. Colon \\
\hline & \multicolumn{4}{|c|}{ Small Intestine } & & Large \\
\hline \multicolumn{6}{|c|}{ Digesta Transit [\%] } & \\
\hline NC & $95.26 \pm 4.01^{\mathrm{ab}}$ & $89.36 \pm 4.58$ & $70.27 \pm 12.07^{\mathrm{a}}$ & $48.53 \pm 4.10^{\mathrm{a} \#}$ & $23.68 \pm 5.63^{\mathrm{a} \#}$ & $36.81 \pm 9.21^{\mathrm{a} \#}$ \\
\hline NSO & $96.46 \pm 1.74^{\mathrm{a} \#}$ & $90.67 \pm 1.34$ & $82.68 \pm 3.70^{b^{*}}$ & $73.93 \pm 2.20^{\mathrm{b}^{*}}$ & $61.61 \pm 4.69^{\mathrm{b}^{* \#}}$ & $68.16 \pm 2.16^{\mathrm{b}^{* \#}}$ \\
\hline DBC & $90.97 \pm 0.08^{\mathrm{b}}$ & $87.86 \pm 1.59$ & $76.55 \pm 5.16^{\mathrm{ab}}$ & $72.01 \pm 7.04^{\mathrm{b}^{*}}$ & $37.56 \pm 3.58^{\mathrm{c}^{*}}$ & $49.69 \pm 0.90^{\mathrm{c}^{*}}$ \\
\hline DSO & $95.89 \pm 1.20^{\mathrm{ab}}$ & $87.37 \pm 3.88$ & $78.43 \pm 3.56^{\mathrm{ab}}$ & $73.04 \pm 5.52^{\mathrm{b}^{*}}$ & $45.98 \pm 5.41^{\mathrm{d}^{* \#}}$ & $62.98 \pm 2.59^{b^{* \#}}$ \\
\hline DBA & $93.85 \pm 4.72^{\mathrm{ab}}$ & $87.01 \pm 5.29$ & $79.48 \pm 6.09^{\mathrm{ab}}$ & $71.76 \pm 5.38^{\mathrm{b}^{*}}$ & $65.26 \pm 5.25^{\mathrm{b} * \#}$ & $87.63 \pm 3.07^{\mathrm{d}^{* \#}}$ \\
\hline
\end{tabular}

Data are presented as mean \pm SD of five to six animals. ${ }^{\text {abcd }}$ Different superscript letters presented in each column for a given segment are significantly different from each other group of animals, * significantly different from NC group and ${ }^{\#}$ significantly different from DBC group $(\mathrm{p}<0.05$; Tukey's HSD post-hoc test, IBM, SPSS, version 21). GRP, Group; NC, normal control; NSO, normal sorbitol; DBC, diabetic control; DSO, diabetic sorbitol, DBA, diabetic acarbose. 


\section{FIGURE CAPTIONS}

Figure 1. Effects of sorbitol on glucose absorption in isolated rat jejunum. Data are presented as mean \pm SD of five replicates of analysis. ${ }^{\text {abc }}$ Different letters presented above the bars for a given concentration means values are significantly different from each other $(\mathrm{p}<0.05$; Tukey's HSD post-hoc test, IBM, SPSS, version 21).

Figure 2. Effects of sorbitol on glucose uptake with or without insulin in isolated rat psoas muscle. Data are presented as mean $\pm \mathrm{SD}$ of triplicate analysis. ${ }^{\text {abc or } x \mathrm{z}}$ Different letters presented above the bars for with or without insulin means values are significantly different from each other ( $\mathrm{p}<0.05$; Tukey's HSD post-hoc test, IBM, SPSS, version 21).

Figure 3. Glucose absorption index (GAI) in the different GIT segments of different animal groups at the end of a 1 hour experimental period. Data are presented as mean \pm SD of five to six

animals. ${ }^{\mathrm{ab}}$ Different letters presented above the bars for a given segment are significantly different from each other group of animals ( $\mathrm{p}<0.05$; Tukey's HSD post-hoc test, IBM, SPSS, version 21). NC, normal control; NSO, normal sorbitol; DBC, diabetic control; DSO, diabetic sorbitol; DBA, diabetic acarbose. 
Figure 4. Effects of sorbitol on gastric emptying in different animal groups at the end of a 1 hour experimental period. Data are presented as mean $\pm \mathrm{SD}$ of five to six animals. ${ }^{\mathrm{abc}}$ Different letters presented above the bars are significantly different from each other group of animals $(\mathrm{p}<0.05$; Tukey's HSD post-hoc test, IBM, SPSS, version 21). NC, normal control; NSO, normal sorbitol; DBC, diabetic control; DSO, diabetic sorbitol, DBA, diabetic acarbose.

Figure 5. Blood glucose increase in the different animal groups 1 hour after dose administration. Data are presented as mean \pm SD of six animals. ${ }^{a b c}$ Different letters presented above the bars for a given segment are significantly different from each other group of animals $(\mathrm{p}<0.05$; Tukey's HSD multiple range post-hoc test). NC, normal control; NSO, normal sorbitol; DBC, diabetic control; DSO, diabetic sorbitol; DBA, diabetic acarbose. 
FIG. 1

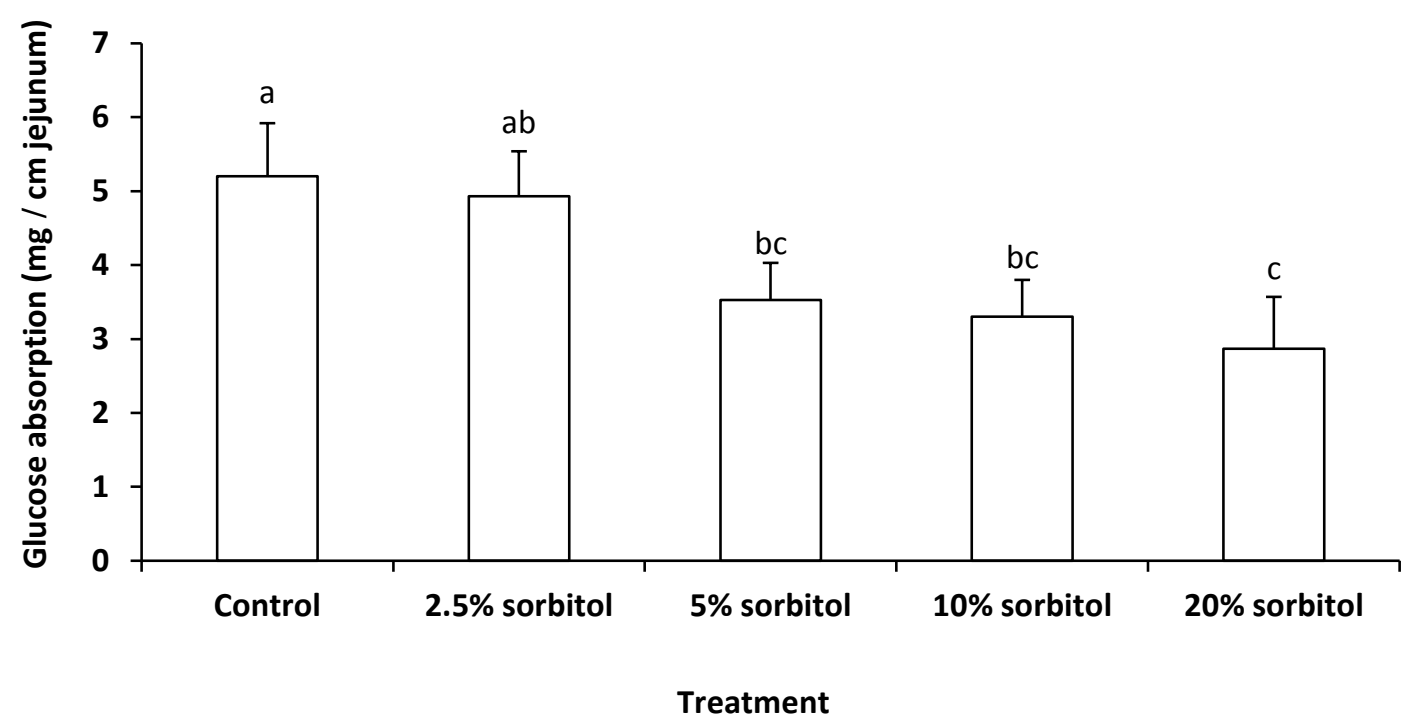


FIG. 2

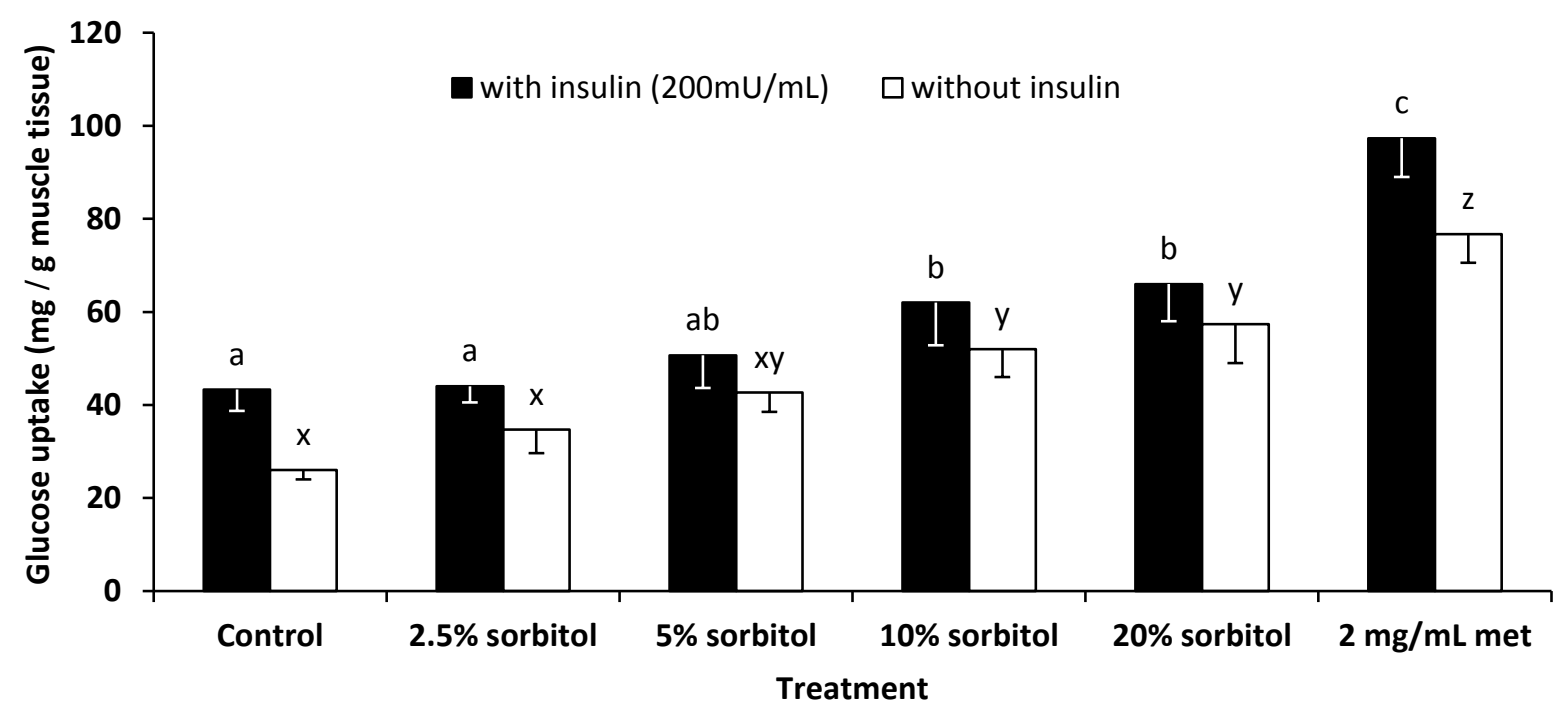


FIG. 3

$\square N C \square N S O \square D B C \square D S O \square D B A$

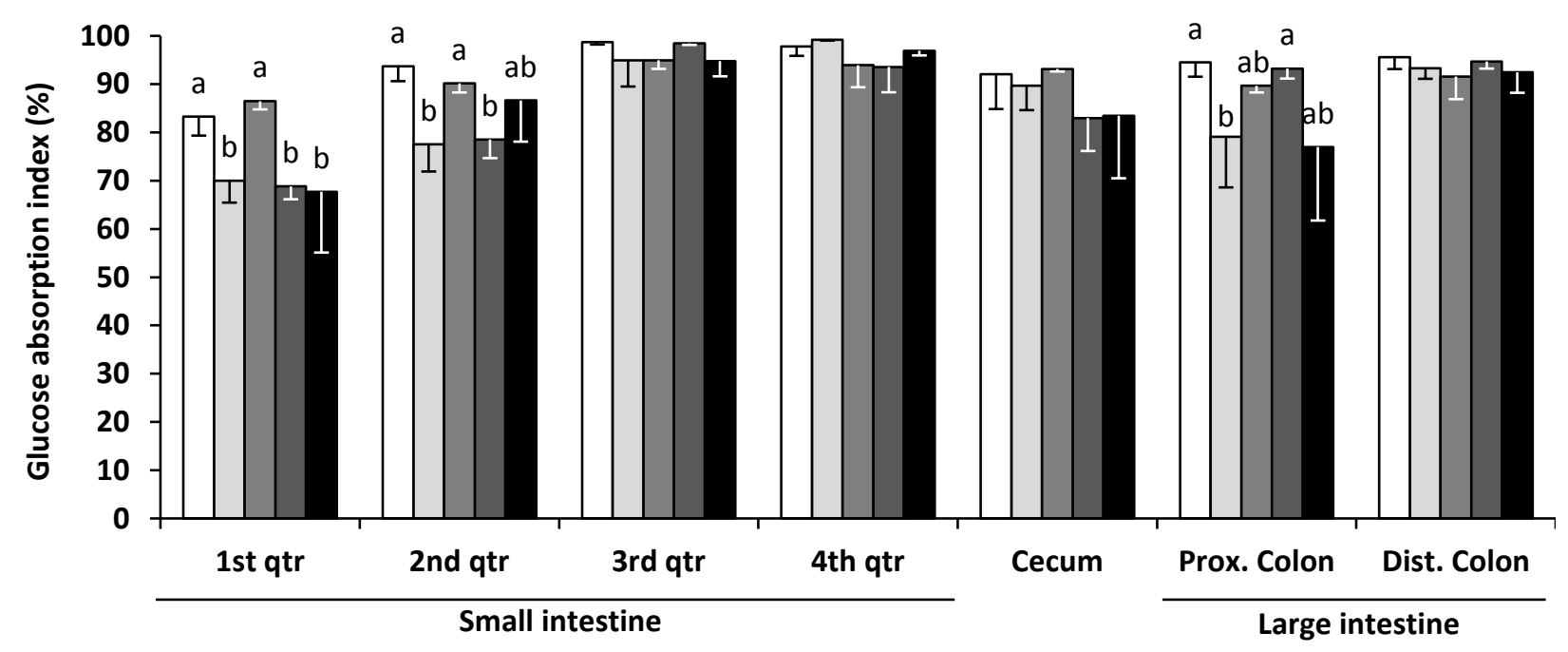


FIG. 4

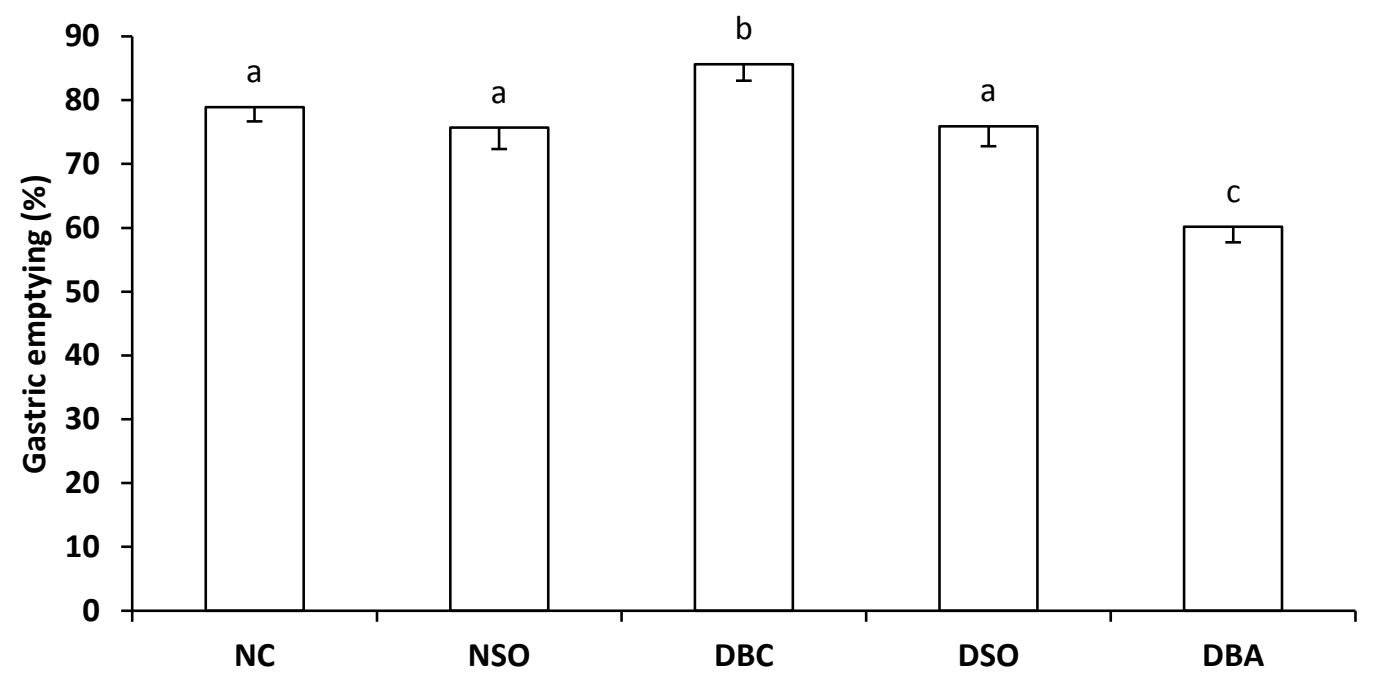


FIG. 5

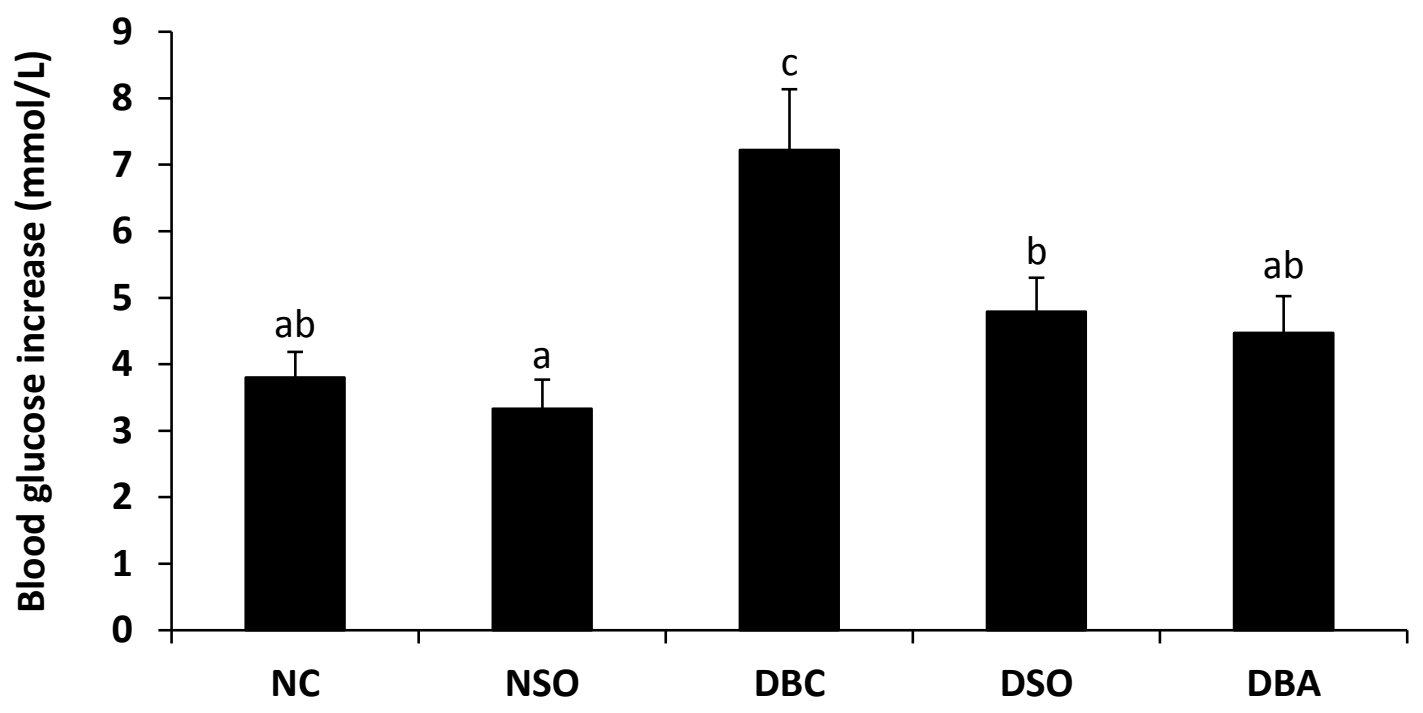

